\title{
The Unruh Effect Revisited
}

\author{
Pietro Longh: \\ Department of Physics and Astronomy \\ Rutgers, The State University of New Jersey \\ 136 Frelinghuysen Road, \\ Piscataway, NJ 08854-8019 USA \\ Roberto Soldat \\ Dipartimento di Fisica, Università di Bologna \\ Istituto Nazionale di Fisica Nucleare, Sezione di Bologna \\ Via Irnerio 46, 40126 - Bologna (Italy)
}

(Dated: March 5, 2018)

\begin{abstract}
A new and exact derivation of the Bogoliubov coefficients is obtained for the simplest case of a spinless, neutral, massive field in a uniformly accelerated frame with a constant acceleration. The method can be suitably generalized in a straightforward manner to any field with spin and charges.
\end{abstract}

PACS numbers: 03.70.+k,98.80.Qc

The so called Unruh effect [1] has been discovered long ago and since its very appearance a really large number of investigations has been developed to the aim of applying and generalizing its deep and seminal content to a wide range of physical contexts, such as e.g. black holes, dark matter and dark energy, cosmological horizons, acoustic black holes et cetera. A very nice and recent review paper [2] on this subject actually provides a pretty good taste of the impressive growth of interest on this subject during the last few years, including the possible occurrence [3] of its experimental observation. From the field theoretical point of view, the Unruh effect is encoded in the Bogoliubov coefficients that relate the complete and orthonormal sets of the wave functions, as experienced by two observers moving respectively in an inertial reference frame and in a noninertial accelerated one. Curiously enough, as reported in [2], the evaluation of the Bogoliubov coefficients in the case of a constant relative acceleration has never been done directly from the very definition but arguing indirectly, sometimes in an admittedly tricky manner [4].

It is the aim of the present note to fill that lack, i.e., we will perform a straightforward exact calculation of the Bogoliubov coefficient from field theoretic first principles. To start with, we briefly review the canonical quantization of a spinless and chargeless field in a Rindler's coordinate system, i.e., as experienced by some uniformly accelerated noninertial observer. In so doing we shall establish our notations and conventions. Consider the four dimensional Minkowski spacetime with the line element [5]

$$
\mathrm{d} s^{2}=\eta_{\alpha \beta} \mathrm{d} X^{\alpha} \mathrm{d} X^{\beta}=g_{\mu \nu}(\mathrm{x}) \mathrm{dx}{ }^{\mu} \mathrm{dx}{ }^{\nu}
$$

where the constant metric tensor $\eta_{\alpha \beta}=\operatorname{diag}(+,-,-,-)$ is relative to an inertial coordinate system in Minkowski

\footnotetext{
* longhi@physics.rutgers.edu

† roberto.soldati@bo.infn.it
}

spacetime and labelled by the so called anholomic indices denoted with the greek letters from the first part of the alphabeth, while the Einstein convention on the sum over repeated indices is understood. We employ natural units $\hbar=c=1$ unless explicitly stated. If we set

$$
X^{\alpha}=(\tau, X, y, z) \quad \mathrm{x}^{\mu}=(t, x, y, z)
$$

then we shall denote the following spacelike region of the Minkowski spacetime

$$
\mathfrak{W}_{R}=\left\{X^{\mu} \in \mathbb{R}^{4} \mid X \geq 0, \tau^{2} \leq X^{2}\right\}
$$

as the right Rindler wedge. Here we introduce the so called Rindler curvilinear coordinate system, which describes an accelerated observer: namely,

$$
\begin{array}{lr}
\tau=x \sinh (\mathrm{a} t) & (\mathrm{a}>0, \xi \in \mathbb{R}) \\
X=x \cosh (\mathrm{a} t) & (x \geq 0)
\end{array}
$$

where a is the constant acceleration. The above coordinate transformations can be readily inverted, viz.,

$$
t=\mathrm{a}^{-1} \operatorname{Arth}(\tau / X) x=\sqrt{X^{2}-\tau^{2}} \quad(x \geq 0)
$$

in such a manner that we can also write

$$
\mathrm{d} s^{2}=g_{\mu \nu}(x) \mathrm{dx}{ }^{\mu} \mathrm{dx}^{\nu}=x^{2} \mathrm{a}^{2} \mathrm{~d} t^{2}-\mathrm{d} x^{2}-\mathrm{d} y^{2}-\mathrm{d} z^{2}
$$

whence we obtain

$$
g_{\mu \nu}(x)=\left(\begin{array}{cccc}
\mathrm{a}^{2} x^{2} & 0 & 0 & 0 \\
0 & -1 & 0 & 0 \\
0 & 0 & -1 & 0 \\
0 & 0 & 0 & -1
\end{array}\right)
$$

so that

$$
g=\operatorname{det} g_{\mu \nu}(x)=\left[\operatorname{det} g^{\mu \nu}(x)\right]^{-1}=-\mathrm{a}^{2} x^{2}
$$

Notice that, by changing both signes in the definitions (3) and (44), we shall cover the other spacelike region of the Minkowski spacetime, i.e. the left Rindler wedge

$$
\mathfrak{W}_{L}=\left\{X^{\mu} \in \mathbb{R}^{4} \mid X \leq 0, \tau^{2} \leq X^{2}\right\}
$$


Moreover we have the Christoffel symbols

$$
\Gamma_{\mu \nu}^{\lambda}(x)=\frac{1}{2} g^{\kappa \lambda}(x)\left\{\partial_{\mu} g_{\nu \kappa}(x)+\partial_{\nu} g_{\mu \kappa}(x)-\partial_{\kappa} g_{\mu \nu}(x)\right\}
$$

so that there are only two nonvanishing components of the affine connection: namely,

$$
\begin{aligned}
& \Gamma_{01}^{0}(x)=\frac{1}{2} g^{00}(x) \frac{\mathrm{d}}{\mathrm{d} x} g_{00}(x)=\frac{1}{x}=\Gamma_{10}^{0}(x) \\
& \Gamma_{00}^{1}(x)=\frac{1}{2} \cdot \frac{\mathrm{d}}{\mathrm{d} x} g^{00}(x)=\mathrm{a}^{2} x
\end{aligned}
$$

This entails the coordinate invariant Klein-Gordon second order differential operator

$$
\begin{gathered}
g^{\mu \nu}(x) D_{\mu} \partial_{\nu}+m^{2}=g^{00}(x)\left(\partial_{t}^{2}-\mathrm{a}^{2} x \partial_{x}\right)-\Delta+m^{2} \\
=\frac{1}{x^{2} \mathrm{a}^{2}} \partial_{t}^{2}-\frac{1}{x} \cdot \partial_{x}-\partial_{x}^{2}-\partial_{y}^{2}-\partial_{z}^{2}+m^{2}
\end{gathered}
$$

where $\Delta$ is the Laplace operator, that leads to the invariant Klein-Gordon equation for a spinless field

$$
\left\{g^{\mu \nu}(x) D_{\mu} \partial_{\nu}+m^{2}\right\} \phi(\mathrm{x})=0
$$

The invariant scalar product between two solutions of the covariant Klein-Gordon field equation is defined by [5]

$$
\left(\phi_{2}, \phi_{1}\right) \equiv \oint_{\Sigma} \phi_{2}^{*}(\mathrm{x}) i \stackrel{\leftrightarrow}{\partial}_{\lambda} \phi_{1}(\mathrm{x}) \mathrm{d} \Sigma^{\lambda}
$$

where $\Sigma$ is a 3-dimensional spacelike hypersurface while

$$
\begin{aligned}
& \mathrm{d} \Sigma^{\lambda} \equiv \frac{1}{6} \varepsilon^{\lambda \mu \nu \rho} \mathrm{d} x_{\mu} \mathrm{d} x_{\nu} \mathrm{d} x_{\rho}(-g)^{-1 / 2} \\
& =\frac{1}{6} \varepsilon^{\lambda \mu \nu \rho} g_{\mu \alpha}(x) g_{\nu \beta}(x) g_{\rho \sigma}(x) \mathrm{d} x^{\alpha} \mathrm{d} x^{\beta} \mathrm{d} x^{\sigma}(-g)^{-1 / 2}
\end{aligned}
$$

is the invariant oriented hypersurface element with $\varepsilon^{0123}=1$. Thus, for the initial time 3-dimensional hypersurface in the right Rindler wedge $\mathfrak{W}_{R}$ we get

$$
\mathrm{d} \Sigma^{0}=-\frac{\theta(x)}{\mathrm{a} x} \mathrm{~d} x \mathrm{~d}^{2} \mathbf{x}_{\perp} \quad \mathrm{d} \Sigma^{\imath}=0 \quad(\imath=1,2,3\lceil 16)
$$

where $\theta(x)$ is the Heaviside step distribution. A complete and orthonormal set of stationary solutions for the KleinGordon equation in the right Rindler wedge is provided by the generalization of the so called Fulling modes [6, 7]

$$
\begin{aligned}
u_{E, \mathbf{k}}\left(t, x, \mathbf{x}_{\perp}\right) & =\frac{1}{2 \pi^{2} \sqrt{ } \mathrm{a}} \sqrt{\sinh \left(\frac{\pi E}{\mathrm{a}}\right)} K_{i E / \mathrm{a}}(\kappa x) \\
& \times \exp \left\{-i E t+i \mathbf{k} \cdot \mathbf{x}_{\perp}\right\}
\end{aligned}
$$

where $K_{\nu}(x)$ is the modified Bessel function. One can fix the normalization constant by the natural requirement

$$
\left(u_{E^{\prime}, \mathbf{k}^{\prime}}, u_{E, \mathbf{k}}\right)=\delta\left(E-E^{\prime}\right) \delta\left(\mathbf{k}-\mathbf{k}^{\prime}\right)
$$

Notice that the orthonormalization relation for the real modified Bessel functions functions can be recast in the suggestive form

$$
\int_{0^{+}}^{\infty} \frac{\mathrm{d} x}{x} K_{i \nu^{\prime}}(\kappa x) K_{i \nu}(\kappa x)=\frac{\pi^{2} \delta\left(\nu-\nu^{\prime}\right)}{2 \nu \sinh (-\pi \nu)}
$$

where $\nu \equiv c E / \hbar$ a. Then we eventually get the complete and orthonormal set of the spinless and chargeless normal modes in the right Rindler wedge $\mathfrak{W}_{R}$ : namely,

$$
\begin{array}{cc}
u_{\boldsymbol{\nu}}\left(t, x, \mathbf{x}_{\perp}\right) & =\frac{1}{2 \pi^{2}} \sqrt{\mathrm{a}^{-1} \sinh (\pi \nu)} K_{i \nu}(\kappa x) \\
& \times \exp \left\{-i \mathrm{a} \nu t+i \mathbf{k} \cdot \mathbf{x}_{\perp}\right\} \\
x>0 \quad \kappa=\sqrt{\mathbf{k}^{2}+m^{2}} \quad \boldsymbol{\nu} \equiv(\nu, \mathbf{k})
\end{array}
$$

which satisfy the invariant orthonormality relations

$$
\left(u_{\boldsymbol{\nu}}, u_{\boldsymbol{\nu}^{\prime}}\right)=\mathrm{a}^{-1} \delta\left(\boldsymbol{\nu}-\boldsymbol{\nu}^{\prime}\right)
$$

According to our definitions, the invariant scalar normal modes (21) have the standard canonical dimensions $\left[u_{E, \mathbf{k}}\right]=\left[u_{\boldsymbol{\nu}}\right]=\sqrt{\mathrm{cm}}=\mathrm{eV}^{-1 / 2}$ in natural units. It follows that the Fulling normal modes expansion of the real scalar quantized field on the right Rindler wedge $\mathfrak{W}_{R}$ reads, in accordance with [2, 6, 7]

$$
\phi_{R}(\mathrm{x})=\int_{-\infty}^{\infty} \mathrm{d} \nu \int \mathrm{d}^{2} \mathbf{k}\left[a_{\nu} u_{\boldsymbol{\nu}}(\mathrm{x})+a_{\nu}^{\dagger} u_{\nu}^{*}(\mathrm{x})\right]
$$

where

$$
\begin{aligned}
& {\left[a_{\boldsymbol{\nu}}, a_{\boldsymbol{\nu}^{\prime}}^{\dagger}\right]=\mathrm{a} \delta\left(\nu-\nu^{\prime}\right) \delta\left(\mathbf{k}-\mathbf{k}^{\prime}\right)} \\
& {\left[a_{\boldsymbol{\nu}}, a_{\boldsymbol{\nu}^{\prime}}\right]=0=\left[a_{\boldsymbol{\nu}}^{\dagger}, a_{\boldsymbol{\nu}^{\prime}}^{\dagger}\right]}
\end{aligned}
$$

in such a way that the quantized real scalar field in the Rindler coordinates has the customary dimensions $[\phi]=$ $\mathrm{eV}$ in natural units, while the operators $\left[a_{\boldsymbol{\nu}}\right]=\mathrm{cm}^{3 / 2}$. On the other side, the quantized real scalar Klein-Gordon field in the Minkowski spacetime has the usual normal mode expansion

$$
\phi_{M}(\tau, \mathbf{X})=\int \mathrm{d} \mathbf{K}\left[\bar{a}_{\mathbf{K}} \bar{u}_{\mathbf{K}}(\tau, \mathbf{X})+\bar{a}_{\mathbf{K}}^{\dagger} \bar{u}_{\mathbf{K}}^{*}(\tau, \mathbf{X})\right]
$$

where $K^{\alpha} \equiv\left(K_{0}, K, \mathbf{K}_{\perp}\right)$ and $K_{0}=\omega_{\mathbf{K}}=\sqrt{\mathbf{K}^{2}+m^{2}}$ whereas

$$
\begin{array}{lr}
\bar{u}_{\mathbf{K}}(\tau, \mathbf{X})=\left[(2 \pi)^{3} 2 \omega_{\mathbf{K}}\right]^{-1 / 2} & \exp \left\{-i K_{\alpha} X^{\alpha}\right\} \\
\left(\bar{u}_{\mathbf{K}}, \bar{u}_{\mathbf{K}^{\prime}}\right)=\delta\left(\mathbf{K}-\mathbf{K}^{\prime}\right) & \left(\forall \mathbf{K}, \mathbf{K}^{\prime} \in \mathbb{R}^{3}\right)
\end{array}
$$

in which the creation and destruction operators satisfy the standard algebra

$$
\begin{aligned}
& {\left[\bar{a}_{\mathbf{K}}, \bar{a}_{\mathbf{P}}^{\dagger}\right]=\delta(\mathbf{K}-\mathbf{P})} \\
& {\left[\bar{a}_{\mathbf{K}}, \bar{a}_{\mathbf{P}}\right]=0 \quad\left[\bar{a}_{\mathbf{K}}^{\dagger}, \bar{a}_{\mathbf{P}}^{\dagger}\right]=0}
\end{aligned}
$$

that leads to the invariant canonical commutation relation

$$
\begin{aligned}
& {\left[\phi_{M}(\tau, \mathbf{X}), \phi_{M}\left(\tau^{\prime}, \mathbf{X}^{\prime}\right)\right]=\frac{1}{i} D\left(\tau-\tau^{\prime}, \mathbf{X}-\mathbf{X}^{\prime} ; m\right)} \\
& =\int \mathrm{d} \mathbf{K}\left\{\bar{u}_{\mathbf{K}}(\tau, \mathbf{X}) \bar{u}_{\mathbf{K}}^{*}\left(\tau^{\prime}, \mathbf{X}^{\prime}\right)-\bar{u}_{\mathbf{K}}^{*}(\tau, \mathbf{X}) \bar{u}_{\mathbf{K}}\left(\tau^{\prime}, \mathbf{X}^{\prime}\right)\right\}
\end{aligned}
$$


where $D(\xi ; m)$ is the invariant Pauli-Jordan distribution, which is real, odd and enjoys the microcausality property

$$
\begin{aligned}
& {\left[\phi_{M}(\tau, \mathbf{X}), \phi_{M}\left(\tau^{\prime}, \mathbf{X}^{\prime}\right)\right]=\left[\phi_{R}(t, \mathbf{x}), \phi_{R}\left(t^{\prime}, \mathbf{x}^{\prime}\right)\right]=0} \\
& \forall X^{\mu}, X^{\prime \mu} \in \mathfrak{W}_{R},\left(X-X^{\prime}\right)^{2}<0 \\
& \lim _{\tau^{\prime} \rightarrow \tau} \frac{i \partial}{\partial \tau}\left[\phi_{M}(\tau, \mathbf{X}), \phi_{M}\left(\tau^{\prime}, \mathbf{X}^{\prime}\right)\right]= \\
& \lim _{\tau^{\prime} \rightarrow \tau} \frac{\partial}{\partial \tau} D\left(\tau-\tau^{\prime}, \mathbf{X}-\mathbf{X}^{\prime} ; m\right)=\delta\left(\mathbf{X}-\mathbf{X}^{\prime}\right)
\end{aligned}
$$

Of course it is apparent that, as far as a quantized scalar field is involved, the canonical commutation relations in the right Rindler wedge are frame independent, even for accelerated noninertial observers since, by definition,

$$
\phi_{M}[\tau(t, x), X(t, x), y, z]=\phi_{R}(\mathrm{x}) \quad \forall \mathrm{x} \in \mathfrak{W}_{R}
$$

Things are different and far more tricky for quantized fields with nonvanishing spin.

We now derive the exact structure of the Bogoliubov coefficients for a neutral spinless field, leading to the well known Unruh's effect. To this purpose, we have to deal with two complete orthonormal sets of solutions of the Klein-Gordon equation (13) in the right Rindler wedge and in the Rindler curvilinear coordinate system: namely,

$$
\begin{aligned}
u_{\boldsymbol{\nu}}(\mathrm{x}) & =\frac{1}{2 \pi^{2}} \sqrt{\mathrm{a}^{-1} \sinh (\pi \nu)} K_{i \nu}(\kappa x) \\
& \times \exp \left\{-i \mathrm{a} \nu t+i \mathbf{k} \cdot \mathbf{x}_{\perp}\right\} \\
\bar{u}_{\mathbf{K}}(\mathrm{x}) & =\left[(2 \pi)^{3} 2 \omega_{\mathbf{K}}\right]^{-\frac{1}{2}} \exp \left\{i \mathbb{k}_{t} x+i \mathbf{K}_{\perp} \cdot \mathbf{x}_{\perp}\right\} \\
\mathbb{k}_{t} \equiv & K \cosh (\mathrm{a} t)-\omega_{\mathbf{K}} \sinh (\mathrm{a} t) \quad[x>0, \nu \in \mathbb{R}]
\end{aligned}
$$

Then a direct substitution into the invariant inner product (14) yields for $E=\mathrm{a} \nu$

$$
\begin{aligned}
\left(\bar{u}_{\mathbf{K}}, u_{\boldsymbol{\nu}}\right) & =\delta\left(\mathbf{K}_{\perp}-\mathbf{k}\right) \exp \{-i E t\} \sqrt{\frac{2 \sinh (\pi \nu)}{(2 \pi \mathrm{a})^{3} \omega_{\mathbf{K}}}} \\
& \times \int_{0^{+}}^{\infty} \frac{\mathrm{d} x}{x} \exp \left\{-i \mathbb{k}_{t} x\right\} K_{i E / \mathrm{a}}(\kappa x)\left(x \dot{\mathbb{k}}_{t}-E\right)
\end{aligned}
$$

where $\dot{\mathbb{k}}_{t}=\mathrm{a} K \sinh (\mathrm{a} t)-\mathrm{a} \omega_{\mathbf{K}} \cosh (\mathrm{a} t)$. However, since the invariant inner product is coordinate time independent by construction, in accordance with the textbook notations [5] we can safely write

$$
\begin{aligned}
\left(\bar{u}_{\mathbf{K}}, u_{\boldsymbol{\nu}}\right) & =-\delta\left(\mathbf{k}-\mathbf{K}_{\perp}\right) \sqrt{\frac{2 \sinh (\pi \nu)}{(2 \pi)^{3} \mathrm{a} \omega_{\mathbf{K}}}} \\
& \times \int_{0^{+}}^{\infty} \frac{\mathrm{d} x}{x} \mathrm{e}^{-i K x}\left(\nu+x \omega_{\mathbf{K}}\right) K_{i \nu}(\kappa x) \\
& \equiv \alpha_{\mathbf{K} \boldsymbol{\nu}}
\end{aligned}
$$

and in a quite similar manner

$$
\begin{aligned}
\left(\bar{u}_{\mathbf{K}}^{*}, u_{\boldsymbol{\nu}}\right) & =-\delta\left(\mathbf{k}+\mathbf{K}_{\perp}\right) \sqrt{\frac{2 \sinh (\pi \nu)}{(2 \pi)^{3} \mathrm{a} \omega_{\mathbf{K}}}} \\
& \times \int_{0^{+}}^{\infty} \frac{\mathrm{d} x}{x} \mathrm{e}^{i K x}\left(\nu-x \omega_{\mathbf{K}}\right) K_{i \nu}(\kappa x) \\
& \equiv \beta_{\mathbf{K} \boldsymbol{\nu}}
\end{aligned}
$$

Now we turn to the calculation of the explicit expressions of the Bogoliubov coefficients in terms of a suitable one parameter representation. From [8] we obtain

$$
\begin{aligned}
\alpha_{\mathbf{K} \boldsymbol{\nu}} & =-\frac{1}{2}\left[\pi \mathrm{a} \omega_{\mathbf{K}} \sinh (\pi \nu)\right]^{-1 / 2} \delta\left(\mathbf{k}-\mathbf{K}_{\perp}\right) \\
& \times\left(\frac{2 \kappa}{\kappa+i K}\right)^{i \nu}\left\{F\left(i \nu, i \nu+\frac{1}{2} ; \frac{1}{2} ; \frac{i K-\kappa}{\kappa+i K}\right)\right. \\
& \left.+\frac{2 \nu}{\omega_{\mathbf{K}}}(\kappa-i K) F\left(1+i \nu, i \nu+\frac{1}{2} ; \frac{3}{2} ; \frac{i K-\kappa}{i K+\kappa}\right)\right\} \\
\beta_{\mathbf{K} \boldsymbol{\nu}} & =-\frac{1}{2}\left[\pi \mathrm{a} \omega_{\mathbf{K}} \sinh (\pi \nu)\right]^{-1 / 2} \delta\left(\mathbf{k}+\mathbf{K}_{\perp}\right) \\
& \times\left(\frac{2 \kappa}{\kappa-i K}\right)^{i \nu}\left\{F\left(i \nu, i \nu+\frac{1}{2} ; \frac{1}{2} ; \frac{i K+\kappa}{i K-\kappa}\right)\right. \\
& \left.-\frac{2 \nu}{\omega_{\mathbf{K}}}(\kappa+i K) F\left(1+i \nu, i \nu+\frac{1}{2} ; \frac{3}{2} ; \frac{i K+\kappa}{i K-\kappa}\right)\right\}
\end{aligned}
$$

where $F(a, b ; c ; z)$ denotes the hypergeometric function. If we introduce the one angular parameter representation according to

$$
\begin{aligned}
& \kappa+i K=\omega_{\mathbf{K}} \mathrm{e}^{i \theta_{\mathbf{K}}} \quad \theta_{\mathbf{K}} \equiv \operatorname{arctg} \frac{K}{\sqrt{ }\left(K_{\perp}^{2}+m^{2}\right)} \\
& \frac{i K+\kappa}{i K-\kappa} \equiv(-1) \mathrm{e}^{2 i \theta}=\left(i \mathrm{e}^{i \theta}\right)^{2} \quad\left(-\frac{\pi}{2}<\theta<\frac{\pi}{2}\right)
\end{aligned}
$$

by making use of the the formulæ

$$
\begin{aligned}
& \Gamma(x) \Gamma\left(x+\frac{1}{2}\right)=\frac{2 \sqrt{\pi}}{2^{2 x}} \Gamma(2 x) \\
& (2 k) !=\left(\frac{1}{2}\right)_{k} k ! 2^{2 k} \quad(2 k+1) !=\left(\frac{3}{2}\right)_{k} k ! 2^{2 k}
\end{aligned}
$$

it is straightforward to get

$$
\begin{aligned}
F\left(i \nu, i \nu+\frac{1}{2} ; \frac{1}{2} ;-\mathrm{e}^{ \pm 2 i \theta}\right) & =\sum_{k=0}^{\infty} \frac{(2 i \nu)_{2 k}}{(2 k) !}\left(i \mathrm{e}^{ \pm i \theta}\right)^{2 k} \\
-2 \nu \mathrm{e}^{ \pm i \theta} F(1+i \nu, i \nu & \left.+\frac{1}{2} ; \frac{3}{2} ;-\mathrm{e}^{ \pm 2 i \theta}\right)= \\
& =\sum_{k=0}^{\infty} \frac{(2 i \nu)_{2 k+1}}{(2 k+1) !}\left(i \mathrm{e}^{ \pm i \theta}\right)^{2 k+1}
\end{aligned}
$$

Thus we are able to obtain exactly the sum of the above series in terms of elementary functions. Consider in fact the identity

$$
\begin{aligned}
F(a, b ; b ; z) & =\sum_{k=0}^{\infty} \frac{(a)_{k}}{k !} z^{k}=(1-z)^{-a} \\
& =\sum_{k=0}^{\infty} \frac{(a)_{2 k}}{(2 k) !} z^{2 k}+\sum_{k=0}^{\infty} \frac{(a)_{2 k+1}}{(2 k+1) !} z^{2 k+1}
\end{aligned}
$$


whence we can readily derive

$$
\begin{array}{r}
\sum_{k=0}^{\infty} \frac{(a)_{2 k}}{(2 k) !} z^{2 k}=\frac{1}{2}\left\{(1-z)^{-a}+(1+z)^{-a}\right\} \\
\sum_{k=1}^{\infty} \frac{(a)_{2 k-1}}{(2 k-1) !} z^{2 k-1}=\frac{1}{2}\left\{(1-z)^{-a}-(1+z)^{-a}\right\}
\end{array}
$$

As a consequence, for $a=2 i \nu$ and $z=(i \kappa \mp K) / \omega_{\mathbf{K}}$, we eventually succeed in extracting the exact Bogoliubov coefficients for a spinless neutral massive field, relating an inertial and an accelerated observers, viz.,

$$
\begin{aligned}
& \alpha_{\mathbf{K}} \boldsymbol{\nu}= \\
& \frac{-\delta\left(\mathbf{k}-\mathbf{K}_{\perp}\right)}{\sqrt{4 \pi \mathrm{a} \omega_{\mathbf{K}} \sinh (\pi \nu)}}\left\{\frac{\omega_{\mathbf{K}}}{\omega_{\mathbf{K}}+K+i \kappa} \sqrt{\frac{2 \kappa}{\kappa+i K}}\right\}^{2 i \nu} \\
& =-\delta\left(\mathbf{k}-\mathbf{K}_{\perp}\right) \frac{\mathrm{e}^{\pi \nu / 2}}{\sqrt{4 \pi \mathrm{a} \omega_{\mathbf{K}} \sinh (\pi \nu)}}\left(\frac{\omega_{\mathbf{K}}-K}{\omega_{\mathbf{K}}+K}\right)^{i \nu / 2} \\
& \beta_{\mathbf{K} \boldsymbol{\nu}}= \\
& \frac{-\delta\left(\mathbf{k}+\mathbf{K}_{\perp}\right)}{\sqrt{4 \pi \mathrm{a} \omega_{\mathbf{K}} \sinh (\pi \nu)}}\left\{\frac{\omega_{\mathbf{K}}}{\omega_{\mathbf{K}}+K-i \kappa} \sqrt{\frac{2 \kappa}{\kappa-i K}}\right\}^{2 i \nu} \\
& =-\delta\left(\mathbf{k}+\mathbf{K}_{\perp}\right) \frac{\mathrm{e}^{-\pi \nu / 2}}{\sqrt{4 \pi \mathrm{a} \omega_{\mathbf{K}} \sinh (\pi \nu)}}\left(\frac{\omega_{\mathbf{K}}-K}{\omega_{\mathbf{K}}+K}\right)^{i \nu / 2}
\end{aligned}
$$

which are in full agreement, up to the Dirac $\delta$-distribution in the tranverse wave vectors, with eq.s (2.107) and (2.108) of the review paper [2]. It follows that, keeping apart the classical volume factors

$$
\Omega_{\mathbf{K}}^{( \pm)}=\delta\left(\mathbf{k} \pm \mathbf{K}_{\perp}\right) \sqrt{\frac{c^{3}}{2 \pi \mathrm{a} \omega_{\mathbf{K}}}}
$$

we can understand the square modulus of the complex dimensionless quantities

$$
\frac{-\mathrm{e}^{ \pm \pi \nu / 2}}{\sqrt{2 \sinh (\pi \nu)}}\left(\frac{\omega_{\mathbf{K}}+K}{\omega_{\mathbf{K}}-K}\right)^{-i \nu / 2}
$$

as nonnegative average numbers. As a matter of fact we set

$$
\mathrm{N}_{\nu} \equiv \frac{e^{2 \pi \nu}}{\mathrm{e}^{2 \pi \nu}-1} \quad \overline{\mathrm{N}}_{\nu} \equiv \frac{1}{\mathrm{e}^{2 \pi \nu}-1}
$$

and if we definitely understand as it is customary $2 \pi \nu=$ $\beta E$, where $\beta=(2 \pi c / \hbar \mathrm{a})$ so that $T=\left(\hbar \mathrm{a} / 2 \pi c k_{\mathrm{B}}\right)$ is the famous Unruh temperature [1], $k_{\mathrm{B}}$ being the Boltzmann constant, we eventually recover the thermal bath which is experienced by the accelerated observer in respect to the inertial one or vice versa. Moreover, from the knowledge of the Bogoliubov coefficients one can immediately obtain the transformation law for the creation and destruction operators

$$
\begin{aligned}
& \bar{a}_{\mathbf{K}}=\sum_{\boldsymbol{\nu}}\left[a_{\boldsymbol{\nu}} \alpha_{\mathbf{K} \boldsymbol{\nu}}-\beta_{\mathbf{K} \boldsymbol{\nu}}^{*} a_{\boldsymbol{\nu}}^{\dagger}\right] \\
& \bar{a}_{\mathbf{K}}^{\dagger}=\sum_{\boldsymbol{\nu}}\left[a_{\boldsymbol{\nu}}^{\dagger} \alpha_{\mathbf{K} \boldsymbol{\nu}}^{*}-\beta_{\mathbf{K} \boldsymbol{\nu}} a_{\boldsymbol{\nu}}\right]
\end{aligned}
$$

where

$$
\sum_{\boldsymbol{\nu}} \equiv \mathrm{a} \int_{-\infty}^{\infty} \mathrm{d} \nu \int \mathrm{d}^{2} k
$$

The above derivation, that concerns the spinless neutral field in the right Rindler wedge, can be trivially extended to the left Rindler wedge. Furthermore, our direct method of evaluation for the Bogoliubov coefficients is well tailored to be successfully generalized mutatis mutandis to fields of higher spin and any charge. Finally, concerning the recent debate [7, 9] on the validity of the Unruh effect, our first principles derivation of the Bogoliubov coefficients definitely endorses the claim of [9], in a manner that is substantially different from Unruh's original trick [1].

\section{ACKNOWLEDGMENTS}

R.S. wishes to acknowledge the support of the Istituto Nazionale di Fisica Nucleare, IS PI13, that contributed to the successful completion of this project.
[1] W. G. Unruh, Phys. Rev. D 14, 870 (Aug 1976).

[2] L. C. B. Crispino, A. Higuchi, and G. E. A. Matsas, Rev. Mod. Phys. 80, 787 (Jul 2008), arXiv:0710.5373 [gr-qc].

[3] R. Balbinot, I. Carusotto, A. Fabbri, and A. Recati, International Journal of Modern Physics D 19, 2371
(2010), arXiv:1005.4000 [gr-qc]

[4] S. Takagi, Progress of Theoretical Physics Supplement 88, 1 (1986).

[5] N. D. Birrell and P. C. W. Davies, Quantum Fields in Curved Space (Cambridge, UK: Cambridge University Press, 1984). 
[6] S. A. Fulling, Phys. Rev. D 7, 2850 (May 1973).

[7] A. M. Fedotov, V. D. Mur, N. B. Narozhny, V. A. Belinskii, and B. M. Karnakov, Physics Letters A 254, 126 (1999)
[8] I. S. Gradshteyn and I. M. Ryzhik, Table of integrals, series and products (New York: Academic Press, 5th ed., 1994).

[9] S. A. Fulling and W. G. Unruh, Phys. Rev. D 70, 048701 (Aug 2004). 